\title{
ON GENERALIZATIONS RELATED TO THE LEFT SIDE OF FEJER'S INEQUALITY VIA FRACTIONAL INTEGRAL OPERATOR
}

\author{
ERHAN SET AND BARIŞ ÇELIKK
}

Received 13 March, 2017

\begin{abstract}
In the paper, firstly, a new fractional integral identity is obtained. Then, some new results related to the left side of Fejér's inequality for differentiable mappings whose derivatives in absolute value are convex via fractional integral operator, using this identity with fundamental inequalities such as Hölder's integral inequality, power-mean inequality and triangle inequality for integral, are presented. The results presented here would provide extensions of those proved in [11].
\end{abstract}

2010 Mathematics Subject Classification: 26A33; 26D10; 26D15

Keywords: Hermite-Hadamard-Fejér inequality, convex function, Hölder inequality, fractional integral operator

\section{INTRODUCTION AND PRELIMINARIES}

Let $f: I \subseteq \mathbb{R} \rightarrow \mathbb{R}$ be a convex function defined on the interval $I$ of real numbers and $a, b \in I$ with $a<b$. The inequality

$$
f\left(\frac{a+b}{2}\right) \leq \frac{1}{b-a} \int_{a}^{b} f(x) d x \leq \frac{f(a)+f(b)}{2}
$$

is well known in the literature as Hermite-Hadamard's inequality (see e.g. [5]). The most well-known inequalities related to the integral mean of a convex function $f$ are the Hermite Hadamard inequalities or its weighted versions, the so-called HermiteHadamard-Fejér inequalities. In [6], Fejér established the following inequality:

Let $f:[a, b] \rightarrow \mathbb{R}$ be convex function. Then the inequality

$$
f\left(\frac{a+b}{2}\right) \int_{a}^{b} g(x) d x \leq \int_{a}^{b} f(x) g(x) d x \leq \frac{a+b}{2} \int_{a}^{b} g(x) d x
$$

holds, where $g:[a, b] \rightarrow \mathbb{R}$ is nonnegative, integrable and symmetric to $\frac{a+b}{2}$.

For some results which generalize, improve, and extend the inequalities (1.1) and (1.2), (see e.g. [7, 10-13]). 
In the following, we will give some necessary definitions and preliminary results which are used and referred to throughout this paper.

Definition 1. Let $f \in L_{1}[a, b]$. The Riemann-Liouville integrals $J_{a+}^{\alpha} f$ and $J_{b-}^{\alpha} f$ of order $\alpha>0$ with $a \geq 0$ are defined by

$$
J_{a+}^{\alpha} f(x)=\frac{1}{\Gamma(\alpha)} \int_{a}^{x}(x-t)^{\alpha-1} f(t) d t, x>a
$$

and

$$
J_{b-}^{\alpha} f(x)=\frac{1}{\Gamma(\alpha)} \int_{x}^{b}(t-x)^{\alpha-1} f(t) d t, \quad x<b
$$

respectively where $\Gamma(\alpha)=\int_{0}^{\infty} e^{-t} u^{\alpha-1} d u$. Here is $J_{a+}^{0} f(x)=J_{b-}^{0} f(x)=f(x)$.

Some recent result and properties concerning the this integral operators can be found in $[2-4,8]$.

In [9], Raina introduced a class of functions defined formally by

$$
\widetilde{F}_{\rho, \lambda}^{\sigma}(x)=\mathcal{F}_{\rho, \lambda}^{\sigma(0), \sigma(1), \ldots}(x)=\sum_{k=0}^{\infty} \frac{\sigma(k)}{\Gamma(\rho k+\lambda)} x^{k} \quad(\rho, \lambda>0 ;|x|<\mathbf{R}),
$$

where the coefficients $\sigma(k)(k \in \mathbb{N}=\mathbb{N} \cup\{0\})$ is a bounded sequence of positive real numbers and $\mathbf{R}$ is the set of real numbers. With the help of (1.3), Raina [9] and Agarwal et al. [1] defined the following left-sided and right-sided fractional integral operators respectively, as follows:

$$
\begin{array}{ll}
\left(\mathscr{g}_{\rho, \lambda, a+; w}^{\sigma} \varphi\right)(x)=\int_{a}^{x}(x-t)^{\lambda-1} \mathscr{F}_{\rho, \lambda}^{\sigma}\left[w(x-t)^{\rho}\right] \varphi(t) d t & (x>a), \\
\left(\mathcal{g}_{\rho, \lambda, b-; w}^{\sigma} \varphi\right)(x)=\int_{x}^{b}(t-x)^{\lambda-1} \mathscr{F}_{\rho, \lambda}^{\sigma}\left[w(t-x)^{\rho}\right] \varphi(t) d t & (x<b),
\end{array}
$$

where $\lambda, \rho>0, w \in \mathbb{R}$ and $\varphi(t)$ is such that the integral on the right side exits.

It is easy to verify that $\mathcal{J}_{\rho, \lambda, a+; w}^{\sigma} \varphi(x)$ and $\mathcal{J}_{\rho, \lambda, b-; w}^{\sigma} \varphi(x)$ are bounded integral operators on $L(a, b)$, if

$$
\mathfrak{M}:=\mathscr{F}_{\rho, \lambda+1}^{\sigma}\left[w(b-a)^{\rho}\right]<\infty .
$$

In fact, for $\varphi \in L(a, b)$, we have

$$
\left\|\mathcal{G}_{\rho, \lambda, a+; w}^{\sigma} \varphi(x)\right\|_{1} \leq \mathfrak{M}(b-a)^{\lambda}\|\varphi\|_{1}
$$

and

where

$$
\left\|\mathcal{I}_{\rho, \lambda, b-; w}^{\sigma} \varphi(x)\right\|_{1} \leq \mathfrak{M}(b-a)^{\lambda}\|\varphi\|_{1}
$$

$$
\|\varphi\|_{p}:=\left(\int_{a}^{b}|\varphi(t)|^{p} d t\right)^{\frac{1}{p}} .
$$


Here, many useful fractional integral operators can be obtained by specializing the coefficient $\sigma(k)$. For instance the classical Riemann-Liouville fractional integrals $J_{a+}^{\alpha}$ and $J_{b-}^{\alpha}$ of order $\alpha$ follow easily by setting $\lambda=\alpha, \sigma(0)=1$ and $w=0$ in (1.4) and (1.5).

Set et al. established a new identity and some Hermite-Hadamard-Fejér type inequalities for differentiable mappings whose derivatives in absolute value are convex via Riemann-Liouville fractional integrals as the following:

Lemma 1 ([11]). Let $f:[a, b] \rightarrow \mathbb{R}$ be a differentiable on mapping $(a, b)$ with $a<$ $b$ and let $g:[a, b] \rightarrow \mathbb{R}$. If $f^{\prime}, g \in L[a, b]$, then the following identity for fractional integrals holds:

$$
\begin{aligned}
& f\left(\frac{a+b}{2}\right)\left[J_{\left(\frac{a+b}{2}\right)-}^{\alpha} g(a)+J_{\left(\frac{a+b}{2}\right)+{ }^{\alpha}}^{\alpha} g(b)\right] \\
& -\left[J_{\left(\frac{a+b}{2}\right)-}^{\alpha}(f g)(a)+J_{\left(\frac{a+b}{2}\right)+}^{\alpha}(f g)(b)\right] \\
& =\frac{1}{\Gamma(\alpha)} \int_{a}^{b} k(t) f^{\prime}(t) d t,
\end{aligned}
$$

where

$$
k(t)= \begin{cases}\int_{a}^{t}(s-a)^{\alpha-1} g(s) d s, & t \in\left[a, \frac{a+b}{2}\right], \\ \int_{b}^{t}(b-s)^{\alpha-1} g(s) d s, & t \in\left[\frac{a+b}{2}, b\right] .\end{cases}
$$

Theorem 1 ([11]). Let $f: I \rightarrow \mathbb{R}$ be a differentiable mapping on $I^{\circ}$ and $f^{\prime} \in$ $L[a, b]$ with $a<b$ and $g:[a, b] \rightarrow \mathbb{R}$ is continuous. If $\left|f^{\prime}\right|$ is convex on $[a, b]$, then the following inequality for fractional integrals holds:

$$
\begin{aligned}
& \mid f\left(\frac{a+b}{2}\right)\left[J_{\left(\frac{a+b}{2}\right)-}^{\alpha} g(a)+J_{\left(\frac{a+b}{2}\right)+}^{\alpha} g(b)\right] \\
& -\left[J_{\left(\frac{a+b}{2}\right)-}^{\alpha}(f g)(a)+J_{\left(\frac{a+b}{2}\right)+}^{\alpha}(f g)(b)\right] \mid \\
& \leq \frac{(b-a)^{\alpha+1}|| g \|_{[a, b], \infty}}{2^{\alpha+1}(\alpha+1) \Gamma(\alpha+1)}\left(\left|f^{\prime}(a)\right|+\left|f^{\prime}(b)\right|\right)
\end{aligned}
$$

with $\alpha>0$.

Theorem 2. ([11]) Let $f: I \rightarrow \mathbb{R}$ be a differentiable mapping on $I^{\circ}$ and $f^{\prime} \in$ $L[a, b]$ with $a<b$ and let $g:[a, b] \rightarrow \mathbb{R}$ is continuous. If $\left|f^{\prime}\right|^{q}$ is convex on $[a, b]$, 
$q \geq 1$, then the following inequality for fractional integrals holds:

$$
\begin{aligned}
& \mid f\left(\frac{a+b}{2}\right)\left[J_{\left(\frac{a+b}{2}\right)-}^{\alpha} g(a)+J_{\left(\frac{a+b}{2}\right)+}^{\alpha} g(b)\right] \\
& -\left[J_{\left(\frac{a+b}{2}\right)-}^{\alpha}(f g)(a)+J_{\left(\frac{a+b}{2}\right)+}^{\alpha}(f g)(b)\right] \mid \\
& \leq \frac{(b-a)^{\alpha+1}|| g \|_{[a, b], \infty}}{2^{\alpha+1+\frac{1}{q}}(\alpha+1)(\alpha+2)^{\frac{1}{q}} \Gamma(\alpha+1)} \\
& \times\left\{\left((\alpha+3)\left|f^{\prime}(a)\right|^{q}+(\alpha+1)\left|f^{\prime}(b)\right|^{q}\right)^{\frac{1}{q}}+\left((\alpha+1)\left|f^{\prime}(a)\right|^{q}+(\alpha+3)\left|f^{\prime}(b)\right|^{q}\right)^{\frac{1}{q}}\right\}
\end{aligned}
$$

with $\alpha>0$.

Theorem 3 ([11]). Let $f: I \rightarrow \mathbb{R}$ be a differentiable mapping on $I^{\circ}$ and $f^{\prime} \in$ $L[a, b]$ with $a<b$ and let $g:[a, b] \rightarrow \mathbb{R}$ is continuous. If $\left|f^{\prime}\right|^{q}$ is convex on $[a, b]$, $q>1$, then the following inequality for fractional integrals holds:

$$
\begin{aligned}
& \mid f\left(\frac{a+b}{2}\right)\left[J_{\left(\frac{a+b}{2}\right)-}^{\alpha} g(a)+J_{\left(\frac{a+b}{2}\right)+}^{\alpha} g(b)\right] \\
& -\left[J_{\left(\frac{a+b}{2}\right)-}^{\alpha}(f g)(a)+J_{\left(\frac{a+b}{2}\right)+}^{\alpha}(f g)(b)\right] \mid \\
& \leq \frac{(b-a)^{\alpha+1}|| g \|_{[a, b], \infty}}{2^{\alpha+1+\frac{2}{q}}(\alpha p+1)^{\frac{1}{p}} \Gamma(\alpha+1)} \\
& \times\left[\left(3\left|f^{\prime}(a)\right|^{q}+\left|f^{\prime}(b)\right|^{q}\right)^{\frac{1}{q}}+\left(\left|f^{\prime}(a)\right|^{q}+3\left|f^{\prime}(b)\right|^{q}\right)^{\frac{1}{q}}\right],
\end{aligned}
$$

where $\frac{1}{p}+\frac{1}{q}=1$.

Motivated by results works done in [1,9,11], in this paper we show that Fejér type inequalities containing the Reimann-Liouville fractional integral operator and given in [11] can be extended to fractional integral operator introduced in [1,9].

\section{MAIN RESULTS}

Lemma 2. Let $f:[a, b] \rightarrow \mathbb{R}$ be differentiable mapping on $(a, b)$ with $a<b$ and let $g:[a, b] \rightarrow \mathbb{R}$. If $f^{\prime}, g \in L[a, b]$, then the following identity for fractional integrals holds:

$$
f\left(\frac{a+b}{2}\right)\left[\mathcal{g}_{\rho, \alpha, \frac{a+b}{2}+; w}^{\sigma} g(b)+\mathcal{g}_{\rho, \alpha, \frac{a+b}{2}-; w}^{\sigma} g(a)\right]
$$




$$
\begin{aligned}
& -\left[\mathcal{g}_{\rho, \alpha, \frac{a+b}{2}+; w}^{\sigma}(f g)(b)+\mathcal{g}_{\rho, \alpha, \frac{a+b}{2}-; w}^{\sigma}(f g)(a)\right] \\
& =\int_{a}^{\frac{a+b}{2}}\left(\int_{a}^{t}(s-a)^{\alpha-1} \mathcal{F}_{\rho, \alpha}^{\sigma}\left[w(s-a)^{\rho}\right] g(s) d s\right) f^{\prime}(t) d t \\
& +\int_{\frac{a+b}{2}}^{b}\left(\int_{b}^{t}(b-s)^{\alpha-1}{\underset{\mathcal{F}}{\rho, \alpha}}_{\sigma, \alpha}^{\sigma}\left[w(b-s)^{\rho}\right] g(s) d s\right) f^{\prime}(t) d t .
\end{aligned}
$$

Proof. It suffices to note that

$$
\begin{aligned}
I & =\int_{a}^{\frac{a+b}{2}}\left(\int_{a}^{t}(s-a)^{\alpha-1} \mathcal{F}_{\rho, \alpha}^{\sigma}\left[w(s-a)^{\rho}\right] g(s) d s\right) f^{\prime}(t) d t \\
& +\int_{\frac{a+b}{2}}^{b}\left(\int_{b}^{t}(b-s)^{\alpha-1} \mathcal{F}_{\rho, \alpha}^{\sigma}\left[w(b-s)^{\rho}\right] g(s) d s\right) f^{\prime}(t) d t \\
& =I_{1}+I_{2} .
\end{aligned}
$$

By integration by parts, we get

$$
\begin{aligned}
I_{1} & =\left.\left(\int_{a}^{t}(s-a)^{\alpha-1} \mathcal{F}_{\rho, \alpha}^{\sigma}\left[w(s-a)^{\rho}\right] g(s) d s\right) f(t) d t\right|_{a} ^{\frac{a+b}{2}} \\
& -\int_{a}^{\frac{a+b}{2}}(t-a)^{\alpha-1} \mathcal{F}_{\rho, \alpha}^{\sigma}\left[w(t-a)^{\rho}\right] g(t) f(t) d t \\
& =\left(\int_{a}^{\frac{a+b}{2}}(s-a)^{\alpha-1} \mathcal{F}_{\rho, \alpha}^{\sigma}\left[w(s-a)^{\rho}\right] g(s) d s\right) f\left(\frac{a+b}{2}\right) \\
& -\int_{a}^{\frac{a+b}{2}}(t-a)^{\alpha-1} \mathcal{F}_{\rho, \alpha}^{\sigma}\left[w(t-a)^{\rho}\right](f g)(t) d t \\
& =f\left(\frac{a+b}{2}\right) \mathscr{g}_{\rho, \alpha, \frac{a+b}{2}-; w}^{\sigma} g(a)-\mathcal{g}_{\rho, \alpha, \frac{a+b}{2}-; w}^{\sigma}(f g)(a)
\end{aligned}
$$

and similarly

$$
\begin{aligned}
I_{2} & =\left.\left(\int_{b}^{t}(b-s)^{\alpha-1} \mathcal{F}_{\rho, \alpha}^{\sigma}\left[w(b-s)^{\rho}\right] g(s) d s\right) f(t) d t\right|_{\frac{a+b}{2}} ^{b} \\
& -\int_{\frac{a+b}{2}}^{b}(b-t)^{\alpha-1} \widetilde{F}_{\rho, \alpha}^{\sigma}\left[w(b-t)^{\rho}\right] g(t) f(t) d t \\
& =\left(\int_{\frac{a+b}{2}}^{b}(b-s)^{\alpha-1}{ }_{\mathcal{F}_{\rho, \alpha}}^{\sigma}\left[w(b-s)^{\rho}\right] g(s) d s\right) f\left(\frac{a+b}{2}\right)
\end{aligned}
$$




$$
\begin{aligned}
& -\int_{\frac{a+b}{2}}^{b}(b-t)^{\alpha-1} \mathcal{F}_{\rho, \alpha}^{\sigma}\left[w(b-t)^{\rho}\right](f g)(t) d t \\
& =f\left(\frac{a+b}{2}\right) \mathcal{g}_{\rho, \alpha, \frac{a+b}{2}+; w}^{\sigma} g(b)-\mathcal{g}_{\rho, \alpha, \frac{a+b}{2}+; w}^{\sigma}(f g)(b) .
\end{aligned}
$$

Putting (2.3) and (2.4) in (2.2), we obtain (2.1) which completes the proof.

Theorem 4. Let $f: I \rightarrow \mathbb{R}$ be a differentiable mapping on $I^{\circ}$ and $f^{\prime} \in L[a, b]$ with $a<b$ and $g:[a, b] \rightarrow \mathbb{R}$ is continuous. If $\left|f^{\prime}\right|$ is convex on $[a, b]$, then the following for fractional integrals holds:

$$
\begin{aligned}
& \mid f\left(\frac{a+b}{2}\right)\left[\mathcal{g}_{\rho, \alpha, \frac{a+b}{2}+; w}^{\sigma} g(b)+\mathcal{g}_{\rho, \alpha, \frac{a+b}{2}-; w}^{\sigma} g(a)\right] \\
& -\left[\mathcal{g}_{\rho, \alpha, \frac{a+b}{2}+; w}^{\sigma}(f g)(b)+\mathcal{g}_{\rho, \alpha, \frac{a+b}{2}-; w}^{\sigma}(f g)(a)\right] \mid \\
& \leq(b-a)^{\alpha+1}\|g\|_{[a, b], \infty} \mathcal{F}_{\rho, \alpha+1}^{\sigma_{1}}\left[|w|(b-a)^{\rho}\right]\left(\left|f^{\prime}(a)\right|+\left|f^{\prime}(b)\right|\right)
\end{aligned}
$$

where

with $\alpha>0$.

$$
\sigma_{1}(k):=\sigma(k) \frac{1}{2^{\alpha+\rho k+1}(\alpha+\rho k+1)}
$$

Proof. Since $\left|f^{\prime}\right|$ is convex on $[a, b]$, we know that for $t \in[a, b]$

$$
\left|f^{\prime}(t)\right|=\left|f^{\prime}\left(\frac{b-t}{b-a} a+\frac{t-a}{b-a} b\right)\right| \leq \frac{b-t}{b-a}\left|f^{\prime}(a)\right|+\frac{t-a}{b-a}\left|f^{\prime}(b)\right| .
$$

From Lemma 2 we have

$$
\begin{aligned}
& \mid f\left(\frac{a+b}{2}\right)\left[\begin{array}{c}
g_{\rho, \alpha, \frac{a+b}{2}}^{\sigma}+; w \\
-
\end{array} g_{\rho, \alpha, \frac{a+b}{2}+; w}^{\sigma}(f g)(b)+\mathcal{g}_{\rho, \alpha, \frac{a+b}{2}-; w}^{\sigma} g(a)\right] \\
& \leq \int_{a}^{\frac{a+b}{2}}\left|\int_{a}^{t}(s-a)^{\alpha-1} \mathcal{F}_{\rho, \alpha}^{\sigma}\left[w(s-a)^{\rho}\right] g(s) d s\right|\left|f^{\prime}(t)\right| d t \\
& +\int_{\frac{a+b}{2}}^{b}\left|\int_{b}^{t}(b-s)^{\alpha-1} \mathcal{F}_{\rho, \alpha}^{\sigma}\left[w(b-s)^{\rho}\right] g(s) d s\right|\left|f^{\prime}(t)\right| d t \\
& \leq \frac{\left.|| g\right|_{\left[a, \frac{a+b}{2}\right], \infty}}{b-a} \int_{a}^{\frac{a+b}{2}}\left(\int_{a}^{t}(s-a)^{\alpha-1}\left(\sum_{k=0}^{\infty} \frac{\sigma(k)|w|^{k}}{\Gamma(\alpha+\rho k)}(s-a)^{\rho k}\right) d s\right) \\
& \times\left[(b-t)\left|f^{\prime}(a)\right|+(t-a)\left|f^{\prime}(b)\right|\right] d t
\end{aligned}
$$




$$
\begin{aligned}
& +\frac{\|g\|_{\left[\frac{a+b}{2}, b\right], \infty}}{b-a} \int_{\frac{a+b}{2}}^{b}\left(\int_{b}^{t}(b-s)^{\alpha-1}\left(\sum_{k=0}^{\infty} \frac{\sigma(k)|w|^{k}}{\Gamma(\alpha+\rho k)}(b-s)^{\rho k}\right) d s\right) \\
& \times\left[(b-t)\left|f^{\prime}(a)\right|+(t-a)\left|f^{\prime}(b)\right|\right] d t \\
& =\frac{\|g\|_{\left[a, \frac{a+b}{2}\right], \infty}}{b-a} \int_{a}^{\frac{a+b}{2}} \sum_{k=0}^{\infty} \frac{\sigma(k)|w|^{k}}{\Gamma(\alpha+\rho k)}\left(\int_{a}^{t}(s-a)^{\alpha+\rho k-1} d s\right) \\
& \times\left[(b-t)\left|f^{\prime}(a)\right|+(t-a)\left|f^{\prime}(b)\right|\right] d t \\
& +\frac{\|g\|_{\left[\frac{a+b}{2}, b\right], \infty}}{b-a} \int_{\frac{a+b}{2}}^{b} \sum_{k=0}^{\infty} \frac{\sigma(k)|w|^{k}}{\Gamma(\alpha+\rho k)}\left(\int_{b}^{t}(b-s)^{\alpha+\rho k-1} d s\right) \\
& \times\left[(b-t)\left|f^{\prime}(a)\right|+(t-a)\left|f^{\prime}(b)\right|\right] d t \\
& =\frac{\|g\|_{\left[a, \frac{a+b}{2}\right], \infty}}{b-a} \\
& \times \sum_{k=0}^{\infty}\left(\int_{a}^{\frac{a+b}{2}}(t-a)^{\alpha+\rho k}\left[(b-t)\left|f^{\prime}(a)\right|+(t-a)\left|f^{\prime}(b)\right|\right] d t\right) \frac{\sigma(k)|w|^{k}}{\Gamma(\alpha+\rho k+1)} \\
& +\frac{\|g\|_{\left[\frac{a+b}{2}, b\right], \infty}}{b-a} \\
& \times \sum_{k=0}^{\infty}\left(\int_{\frac{a+b}{2}}^{b}(b-t)^{\alpha+\rho k}\left[(b-t)\left|f^{\prime}(a)\right|+(t-a)\left|f^{\prime}(b)\right|\right] d t\right) \frac{\sigma(k)|w|^{k}}{\Gamma(\alpha+\rho k+1)} \\
& =\frac{\|g\|_{\left[a, \frac{a+b}{2}\right], \infty}}{b-a} \\
& \times\left\{\sum _ { k = 0 } ^ { \infty } \frac { \sigma ( k ) | w | ^ { k } } { \Gamma ( \alpha + \rho k + 1 ) } \left[\left(\frac{(\alpha+\rho k+3)(b-a)^{\alpha+\rho k+2}}{2^{\alpha+\rho k+2}(\alpha+\rho k+1)(\alpha+\rho k+2)}\right)\left|f^{\prime}(a)\right|\right.\right. \\
& \left.\left.+\left(\frac{(b-a)^{\alpha+\rho k+2}}{2^{\alpha+\rho k+2}(\alpha+\rho k+2)}\right)\left|f^{\prime}(b)\right|\right]\right\} \\
& +\frac{\|g\|_{\left[\frac{a+b}{2}, b\right], \infty}}{b-a} \\
& \times\left\{\sum _ { k = 0 } ^ { \infty } \frac { \sigma ( k ) | w | ^ { k } } { \Gamma ( \alpha + \rho k + 1 ) } \left[\left(\frac{(b-a)^{\alpha+\rho k+2}}{2^{\alpha+\rho k+2}(\alpha+\rho k+2)}\right)\left|f^{\prime}(a)\right|\right.\right. \\
& \left.\left.+\left(\frac{(\alpha+\rho k+3)(b-a)^{\alpha+\rho k+2}}{2^{\alpha+\rho k+2}(\alpha+\rho k+1)(\alpha+\rho k+2)}\right)\left|f^{\prime}(b)\right|\right]\right\}
\end{aligned}
$$




$$
\begin{aligned}
& \leq \frac{\|g\|_{[a, b], \infty}}{b-a}\left\{\sum_{k=0}^{\infty} \frac{\sigma(k)|w|^{k}}{\Gamma(\alpha+\rho k+1)} \frac{(b-a)^{\alpha+\rho k+2}}{2^{\alpha+\rho k+1}(\alpha+\rho k+1)}\left(\left|f^{\prime}(a)\right|+\left|f^{\prime}(b)\right|\right)\right\} \\
& =\|g\|_{[a, b], \infty}(b-a)^{\alpha+1}{ }_{\mathcal{F}_{\rho, \alpha+1}}^{\sigma_{1}}\left[w(b-a)^{\rho}\right]\left(\left|f^{\prime}(a)\right|+\left|f^{\prime}(b)\right|\right),
\end{aligned}
$$

where

$$
\begin{gathered}
\int_{a}^{\frac{a+b}{2}}(t-a)^{\alpha+\rho k+1} d t=\int_{\frac{a+b}{2}}^{b}(b-t)^{\alpha+\rho k+1} d t=\frac{(b-a)^{\alpha+\rho k+2}}{2^{\alpha+\rho k+2}(\alpha+\rho k+2)} \\
\int_{a}^{\frac{a+b}{2}}(t-a)^{\alpha+\rho k}(b-t) d t=\int_{\frac{a+b}{2}}^{b}(b-t)^{\alpha+\rho k}(t-a) d t \\
=\frac{(\alpha+\rho k+3)(b-a)^{\alpha+\rho k+2}}{2^{\alpha+\rho k+2}(\alpha+\rho k+1)(\alpha+\rho k+2)} .
\end{gathered}
$$

This completes the proof.

Remark 1. If we choose $\sigma(0)=1$ and $w=0$ in Theorem 4 , then the inequality (2.5) reduces to (1.10).

Theorem 5. Let $f: I \rightarrow \mathbb{R}$ be a differentiable mapping on $I^{\circ}$ and $f^{\prime} \in L[a, b]$ with $a<b$ and let $g:[a, b] \rightarrow \mathbb{R}$ is continuous. If $\left|f^{\prime}\right|^{q}$ is convex on $[a, b], q \geq 1$, then the following inequality for fractional integrals holds:

$$
\begin{aligned}
& \mid f\left(\frac{a+b}{2}\right)\left[g_{\rho, \alpha, \frac{a+b}{2}+; w}^{\sigma} g(b)+\mathcal{g}_{\rho, \alpha, \frac{a+b}{2}-; w}^{\sigma} g(a)\right] \\
& -\left[g_{\rho, \alpha, \frac{a+b}{2}+; w}^{\sigma}(f g)(b)+g_{\rho, \alpha, \frac{a+b}{2}-; w}^{\sigma}(f g)(a)\right] \mid \\
& \leq|| g \|_{[a, b], \infty}\left((b-a)^{\alpha+1}\right)^{\frac{1}{q}}\left((b-a)^{\alpha+1} \mathcal{F}_{\rho, \alpha+1}^{\sigma_{1}}\left[|w|(b-a)^{\rho}\right]\right)^{1-\frac{1}{q}} \\
& \times\left\{\left[\mathcal{F}_{\rho, \alpha+1}^{\sigma_{2}}\left[|w|(b-a)^{\rho}\right]\left|f^{\prime}(a)\right|^{q}+\mathcal{F}_{\rho, \alpha+1}^{\sigma_{3}}\left[|w|(b-a)^{\rho}\right]\left|f^{\prime}(b)\right|^{q}\right]^{\frac{1}{q}}\right. \\
& \left.+\left[\mathcal{F}_{\rho, \alpha+1}^{\sigma_{3}}\left[|w|(b-a)^{\rho}\right]\left|f^{\prime}(a)\right|^{q}+\mathcal{F}_{\rho, \alpha+1}^{\sigma_{2}}\left[|w|(b-a)^{\rho}\right]\left|f^{\prime}(b)\right|^{q}\right]^{\frac{1}{q}}\right\}
\end{aligned}
$$

where

$$
\begin{gathered}
\sigma_{1}(k):=\sigma(k) \frac{1}{(\alpha+\rho k+1) 2^{\alpha+\rho k+1}}, \\
\sigma_{2}(k):=\sigma(k) \frac{\alpha+\rho k+3}{(\alpha+\rho k+1)(\alpha+\rho k+2) 2^{\alpha+\rho k+2}}
\end{gathered}
$$

and

$$
\sigma_{3}(k):=\sigma(k) \frac{1}{(\alpha+\rho k+2) 2^{\alpha+\rho k+2}}
$$


with $\alpha>0$.

Proof. Since $\left|f^{\prime}\right|^{q}$ is convex on $[a, b]$, we know that for $t \in[a, b]$

$$
\left|f^{\prime}(t)\right|^{q}=\left|f^{\prime}\left(\frac{b-t}{b-a} a+\frac{t-a}{b-a} b\right)\right|^{q} \leq \frac{b-t}{b-a}\left|f^{\prime}(a)\right|^{q}+\frac{t-a}{b-a}\left|f^{\prime}(b)\right|^{q} .
$$

Using Lemma 2, power-mean inequality and the convexity of $\left|f^{\prime}\right|^{q}$, it follows that

$$
\begin{aligned}
& f\left(\frac{a+b}{2}\right)\left[\mathcal{g}_{\rho, \alpha, \frac{a+b}{2}+; w}^{\sigma} g(b)+\mathcal{g}_{\rho, \alpha, \frac{a+b}{2}-; w}^{\sigma} g(a)\right] \\
& -\left[\mathcal{g}_{\rho, \alpha, \frac{a+b}{2}+; w}^{\sigma}(f g)(b)+\mathcal{g}_{\rho, \alpha, \frac{a+b}{2}-; w}^{\sigma}(f g)(a)\right] \mid \\
& \leq\left(\int_{a}^{\frac{a+b}{2}}\left|\int_{a}^{t}(s-a)^{\alpha-1} \widetilde{F}_{\rho, \alpha}^{\sigma}\left[w(s-a)^{\rho}\right] g(s) d s\right| d t\right)^{1-\frac{1}{q}} \\
& \times\left(\int_{a}^{\frac{a+b}{2}}\left|\int_{a}^{t}(s-a)^{\alpha-1} \mathcal{F}_{\rho, \alpha}^{\sigma}\left[w(b-a)^{\rho}\right] g(s) d s\right|\left|f^{\prime}(t)\right|^{q} d t\right)^{\frac{1}{q}} \\
& +\left(\int_{\frac{a+b}{2}}^{b}\left|\int_{b}^{t}(b-s)^{\alpha-1} \mathcal{F}_{\rho, \alpha}^{\sigma}\left[w(b-s)^{\rho}\right] g(s) d s\right| d t\right)^{1-\frac{1}{q}} \\
& \times\left(\int_{\frac{a+b}{2}}^{b}\left|\int_{b}^{t}(b-s)^{\alpha-1} \mathcal{F}_{\rho, \alpha}^{\sigma}\left[w(b-s)^{\rho}\right] g(s) d s\right|\left|f^{\prime}(t)\right|^{q} d t\right)^{\frac{1}{q}} \\
& \leq\|g\|_{\left[a, \frac{a+b}{2}\right], \infty}\left(\int_{a}^{\frac{a+b}{2}} \sum_{k=0}^{\infty} \frac{\sigma(k)|w|^{k}}{\Gamma(\alpha+\rho k)}\left|\int_{a}^{t}(s-a)^{\alpha+\rho k-1} d s\right| d t\right)^{1-\frac{1}{q}} \\
& \times\left(\int_{a}^{\frac{a+b}{2}} \sum_{k=0}^{\infty} \frac{\sigma(k)|w|^{k}}{\Gamma(\alpha+\rho k)}\left|\int_{a}^{t}(s-a)^{\alpha+\rho k-1} d s\right|\left|f^{\prime}(t)\right|^{q} d t\right)^{\frac{1}{q}} \\
& +\|g\|_{\left[\frac{a+b}{2}, b\right], \infty}\left(\int_{\frac{a+b}{2}}^{b} \sum_{k=0}^{\infty} \frac{\sigma(k)|w|^{k}}{\Gamma(\alpha+\rho k)}\left|\int_{b}^{t}(b-s)^{\alpha+\rho k-1} d s\right| d t\right)^{1-\frac{1}{q}} \\
& \times\left(\int_{\frac{a+b}{2}}^{b} \sum_{k=0}^{\infty} \frac{\sigma(k)|w|^{k}}{\Gamma(\alpha+\rho k)}\left|\int_{b}^{t}(b-s)^{\alpha+\rho k-1} d s\right|\left|f^{\prime}(t)\right|^{q} d t\right)^{\frac{1}{q}} \\
& \leq\left((b-a)^{\alpha+1} \sum_{k=0}^{\infty} \frac{\sigma(k)|w|^{k}}{\Gamma(\alpha+\rho k)} \frac{(b-a)^{\rho k}}{(\alpha+\rho k)(\alpha+\rho k+1) 2^{\alpha+\rho k+1}}\right)^{1-\frac{1}{q}}
\end{aligned}
$$




$$
\begin{aligned}
& \times\left\{\frac { \| g \| _ { [ a , \frac { a + b } { 2 } ] , \infty } } { ( b - a ) ^ { \frac { 1 } { q } } } \left(\int_{a}^{\frac{a+b}{2}} \sum_{k=0}^{\infty} \frac{\sigma(k)|w|^{k}}{\Gamma(\alpha+\rho k)(\alpha+\rho k)}\right.\right. \\
& \left.\times\left[(t-a)^{\alpha+\rho k}(b-t)\left|f^{\prime}(a)\right|^{q}+(t-a)^{\alpha+\rho k+1}\left|f^{\prime}(b)\right|^{q}\right] d t\right)^{\frac{1}{q}} \\
& +\frac{\|g\|_{\left[\frac{a+b}{2}, b\right], \infty}}{(b-a)^{\frac{1}{q}}}\left(\int_{\frac{a+b}{2}}^{b} \sum_{k=0}^{\infty} \frac{\sigma(k)|w|^{k}}{\Gamma(\alpha+\rho k)(\alpha+\rho k)}\right. \\
& \left.\left.\times\left[(b-t)^{\alpha+\rho k+1}\left|f^{\prime}(a)\right|^{q}+(b-t)^{\alpha+\rho k}(t-a)\left|f^{\prime}(b)\right|^{q}\right] d t\right)^{\frac{1}{q}}\right\} \\
& =\left((b-a)^{\alpha+1}{ }_{\mathcal{F}}^{\sigma_{\rho, \alpha+1}}\left[w(b-a)^{\rho}\right]\right)^{1-\frac{1}{q}} \\
& \times\left\{\frac { \| g \| _ { [ a , \frac { a + b } { 2 } ] , \infty } } { ( b - a ) ^ { \frac { 1 } { q } } } \left(\sum _ { k = 0 } ^ { \infty } \left(\frac{\sigma(k)|w|^{k}}{\Gamma(\alpha+\rho k)(\alpha+\rho k)}\right.\right.\right. \\
& \times\left[\frac{(\alpha+\rho k+3)(b-a)^{\alpha+\rho k+2}}{2^{\alpha+\rho k+2}(\alpha+\rho k+1)(\alpha+\rho k+2)}\left|f^{\prime}(a)\right|^{q}\right. \\
& \left.\left.\left.+\frac{(b-a)^{\alpha+\rho k+2}}{2^{\alpha+\rho k+2}(\alpha+\rho k+2)}\left|f^{\prime}(b)\right|^{q}\right]\right)\right)^{\frac{1}{q}} \\
& +\frac{\|g\|_{\left[\frac{a+b}{2}, b\right], \infty}}{(b-a)^{\frac{1}{q}}}\left(\sum _ { k = 0 } ^ { \infty } \left(\frac{\sigma(k)|w|^{k}}{\Gamma(\alpha+\rho k)(\alpha+\rho k)}\right.\right. \\
& \times\left[\frac{(b-a)^{\alpha+\rho k+2}}{2^{\alpha+\rho k+2}(\alpha+\rho k+2)}\left|f^{\prime}(a)\right|^{q}\right. \\
& \left.\left.\left.\left.+\frac{(\alpha+\rho k+3)(b-a)^{\alpha+\rho k+2}}{2^{\alpha+\rho k+2}(\alpha+\rho k+1)(\alpha+\rho k+2)}\left|f^{\prime}(b)\right|^{q}\right]\right)\right)^{\frac{1}{q}}\right\} \\
& \leq\|g\|_{[a, b], \infty}\left((b-a)^{\alpha+1}\right)^{\frac{1}{q}}\left((b-a)^{\alpha+1} \mathcal{F}_{\rho, \alpha+1}^{\sigma_{1}}\left[|w|(b-a)^{\rho}\right]\right)^{1-\frac{1}{q}} \\
& \times\left\{\left[\mathcal{F}_{\rho, \alpha+1}^{\sigma_{2}}\left[|w|(b-a)^{\rho}\right]\left|f^{\prime}(a)\right|^{q}+\mathcal{F}_{\rho, \alpha+1}^{\sigma_{3}}\left[|w|(b-a)^{\rho}\right]\left|f^{\prime}(b)\right|^{q}\right]^{\frac{1}{q}}\right. \\
& \left.+\left[\mathscr{F}_{\rho, \alpha+1}^{\sigma_{3}}\left[|w|(b-a)^{\rho}\right]\left|f^{\prime}(a)\right|^{q}+\mathscr{F}_{\rho, \alpha+1}^{\sigma_{2}}\left[|w|(b-a)^{\rho}\right]\left|f^{\prime}(b)\right|^{q}\right]^{\frac{1}{q}}\right\},
\end{aligned}
$$


where it is easily seen that

$$
\begin{aligned}
\int_{a}^{\frac{a+b}{2}}\left|\int_{a}^{t}(s-a)^{\alpha+\rho k-1} d s\right| d t & =\int_{\frac{a+b}{2}}^{b}\left|\int_{b}^{t}(b-s)^{\alpha+\rho k-1} d s\right| d t \\
& =\frac{(b-a)^{\alpha+\rho k+1}}{2^{\alpha+\rho k+1}(\alpha+\rho k)(\alpha+\rho k+1)} \\
\int_{a}^{\frac{a+b}{2}}(t-a)^{\alpha+\rho k}(b-t) d t & =\int_{\frac{a+b}{2}}^{b}(b-t)^{\alpha+\rho k}(t-a) d t \\
& =\frac{(\alpha+\rho k+3)(b-a)^{\alpha+\rho k+2}}{2^{\alpha+\rho k+2}(\alpha+\rho k+1)(\alpha+\rho k+2)} .
\end{aligned}
$$

Hence the proof is completed.

Remark 2. If we choose $\sigma(0)=1$ and $w=0$ in Theorem 5, then the inequality (2.6) reduces to (1.11).

Theorem 6. Let $f: I \rightarrow \mathbb{R}$ be a differentiable mapping on $I^{\circ}$ and $f^{\prime} \in L[a, b]$ with $a<b$ and let $g:[a, b] \rightarrow \mathbb{R}$ is continuous. If $\left|f^{\prime}\right|^{q}$ is convex on $[a, b], q \geq 1$, then the following inequality for fractional integrals holds:

$$
\begin{aligned}
& \mid f\left(\frac{a+b}{2}\right)\left[g_{\rho, \alpha, \frac{a+b}{2}+; w}^{\sigma} g(b)+\mathcal{g}_{\rho, \alpha, \frac{a+b}{2}-; w}^{\sigma} g(a)\right] \\
& -\left[g_{\rho, \alpha, \frac{a+b}{2}+; w}^{\sigma}(f g)(b)+g_{\rho, \alpha, \frac{a+b}{2}-; w}^{\sigma}(f g)(a)\right] \mid \\
& \leq \frac{(b-a)^{\alpha+1}|| g \|_{[a, b]_{\infty}}}{2^{\frac{3}{q}}}\left(\mathcal{F}_{\rho, \alpha+1}^{\sigma_{1}}\left[|w|(b-a)^{\rho}\right]\right)^{\frac{1}{p}} \\
& \times\left\{\left[3\left|f^{\prime}(a)\right|^{q}+\left|f^{\prime}(b)\right|^{q}\right]^{\frac{1}{q}}+\left[\left|f^{\prime}(a)\right|^{q}+3\left|f^{\prime}(b)\right|^{q}\right]^{\frac{1}{q}}\right\}
\end{aligned}
$$

where

$$
\sigma_{1}(k):=\sigma(k) \frac{1}{2^{\alpha+\rho k+\frac{1}{p}}(\alpha p+\rho k p+1)^{\frac{1}{p}}}
$$

and $\frac{1}{p}+\frac{1}{q}=1$.

Proof. Using Lemma 2, Hölder's inequality and the convexity of $\left|f^{\prime}\right|^{q}$, it follows that

$$
\begin{aligned}
& \mid f\left(\frac{a+b}{2}\right)\left[\mathcal{g}_{\rho, \alpha, \frac{a+b}{2}+; w}^{\sigma} g(b)+\mathcal{g}_{\rho, \alpha, \frac{a+b}{2}-; w}^{\sigma} g(a)\right] \\
& -\left[\mathcal{g}_{\rho, \alpha, \frac{a+b}{2}+; w}^{\sigma}(f g)(b)+\mathcal{g}_{\rho, \alpha, \frac{a+b}{2}-; w}^{\sigma}(f g)(a)\right] \mid
\end{aligned}
$$




$$
\begin{aligned}
& \leq \mid \int_{a}^{\frac{a+b}{2}}\left(\int_{a}^{t}(s-a)^{\alpha-1} \mathcal{F}_{\rho, \alpha}^{\sigma}\left[w(s-a)^{\rho}\right] g(s) d s\right) f^{\prime}(t) d t \\
& +\int_{\frac{a+b}{2}}^{b}\left(\int_{b}^{t}(b-s)^{\alpha-1} \mathcal{F}_{\rho, \alpha}^{\sigma}\left[w(b-s)^{\rho}\right] g(s) d s\right) f^{\prime}(t) d t \mid \\
& \leq \int_{a}^{\frac{a+b}{2}}\left|\int_{a}^{t}(s-a)^{\alpha-1} \mathcal{F}_{\rho, \alpha}^{\sigma}\left[w(s-a)^{\rho}\right] g(s) d s\right|\left|f^{\prime}(t)\right| d t \\
& +\int_{\frac{a+b}{2}}^{b}\left|\int_{b}^{t}(b-s)^{\alpha-1} \mathcal{F}_{\rho, \alpha}^{\sigma}\left[w(b-s)^{\rho}\right] g(s) d s\right|\left|f^{\prime}(t)\right| d t \\
& =\int_{a}^{\frac{a+b}{2}}\left|\int_{a}^{t}(s-a)^{\alpha-1} \sum_{k=0}^{\infty} \frac{\sigma(k) w^{k}(s-a)^{\rho k}}{\Gamma(\alpha+\rho k)} g(s) d s\right|\left|f^{\prime}(t)\right| d t \\
& +\int_{\frac{a+b}{2}}^{b}\left|\int_{b}^{t}(b-s)^{\alpha-1} \sum_{k=0}^{\infty} \frac{\sigma(k) w^{k}(b-s)^{\rho k}}{\Gamma(\alpha+\rho k)} g(s) d s\right|\left|f^{\prime}(t)\right| d t
\end{aligned}
$$

$=\int_{a}^{\frac{a+b}{2}}\left|\sum_{k=0}^{\infty} \frac{\sigma(k) w^{k}}{\Gamma(\alpha+\rho k)} \int_{a}^{t}(s-a)^{\alpha+\rho k-1} g(s) d s\right|\left|f^{\prime}(t)\right| d t$

$+\int_{\frac{a+b}{2}}^{b}\left|\sum_{k=0}^{\infty} \frac{\sigma(k) w^{k}}{\Gamma(\alpha+\rho k)} \int_{b}^{t}(b-s)^{\alpha+\rho k-1} g(s) d s\right|\left|f^{\prime}(t)\right| d t$

$\leq \int_{a}^{\frac{a+b}{2}}\left(\sum_{k=0}^{\infty} \frac{\sigma(k)|w|^{k}}{\Gamma(\alpha+\rho k)}\left|\int_{a}^{t}(s-a)^{\alpha+\rho k-1} g(s) d s\right|\right)\left|f^{\prime}(t)\right| d t$

$+\int_{\frac{a+b}{2}}^{b}\left(\sum_{k=0}^{\infty} \frac{\sigma(k)|w|^{k}}{\Gamma(\alpha+\rho k)}\left|\int_{b}^{t}(b-s)^{\alpha+\rho k-1} g(s) d s\right|\right)\left|f^{\prime}(t)\right| d t$

$\leq\|g\|_{\left[a, \frac{a+b}{2}\right], \infty} \sum_{k=0}^{\infty} \frac{\sigma(k)|w|^{k}}{\Gamma(\alpha+\rho k)}\left(\int_{a}^{\frac{a+b}{2}}\left|\int_{a}^{t}(s-a)^{\alpha+\rho k-1} d s\right|\left|f^{\prime}(t)\right| d t\right)$

$+\|g\|_{\left[\frac{a+b}{2}, b\right], \infty} \sum_{k=0}^{\infty} \frac{\sigma(k)|w|^{k}}{\Gamma(\alpha+\rho k)}\left(\int_{\frac{a+b}{2}}^{b}\left|\int_{b}^{t}(b-s)^{\alpha+\rho k-1} d s\right|\left|f^{\prime}(t)\right| d t\right)$

$\leq\|g\|_{[a, b], \infty}\left\{\sum_{k=0}^{\infty} \frac{\sigma(k)|w|^{k}}{\Gamma(\alpha+\rho k)}\left(\int_{a}^{\frac{a+b}{2}}\left|\int_{a}^{t}(s-a)^{\alpha+\rho k-1} d s\right|^{p} d t\right)^{\frac{1}{p}}\left(\int_{a}^{\frac{a+b}{2}}\left|f^{\prime}(t)\right|^{q} d t\right)^{\frac{1}{q}}\right.$

$\left.+\sum_{k=0}^{\infty} \frac{\sigma(k)|w|^{k}}{\Gamma(\alpha+\rho k)}\left(\int_{\frac{a+b}{2}}^{b}\left|\int_{b}^{t}(b-s)^{\alpha+\rho k-1} d s\right|^{p} d t\right)^{\frac{1}{p}}\left(\int_{\frac{a+b}{2}}^{b}\left|f^{\prime}(t)\right|^{q} d t\right)^{\frac{1}{q}}\right\}$ 
$=\|g\|_{[a, b], \infty}\left\{\sum_{k=0}^{\infty} \frac{\sigma(k)|w|^{k}}{\Gamma(\alpha+\rho k)}\left(\left.\int_{a}^{\frac{a+b}{2}}\left|\frac{(s-a)^{\alpha+\rho k}}{(\alpha+\rho k)}\right|_{a}^{t}\right|^{p} d t\right)^{\frac{1}{p}}\left(\int_{a}^{\frac{a+b}{2}}\left|f^{\prime}(t)\right|^{q} d t\right)^{\frac{1}{q}}\right.$

$\left.+\sum_{k=0}^{\infty} \frac{\sigma(k)|w|^{k}}{\Gamma(\alpha+\rho k)}\left(\left.\int_{\frac{a+b}{2}}^{b}\left|\frac{(b-s)^{\alpha+\rho k}}{(\alpha+\rho k)}\right|_{b}^{t}\right|^{p} d t\right)^{\frac{1}{p}}\left(\int_{\frac{a+b}{2}}^{b}\left|f^{\prime}(t)\right|^{q} d t\right)^{\frac{1}{q}}\right\}$

$=\|g\|_{[a, b], \infty}\left\{\sum_{k=0}^{\infty} \frac{\sigma(k)|w|^{k}}{\Gamma(\alpha+\rho k)}\left(\int_{a}^{\frac{a+b}{2}} \frac{(t-a)^{(\alpha+\rho k) p}}{(\alpha+\rho k)^{p}} d t\right)^{\frac{1}{p}}\left(\int_{a}^{\frac{a+b}{2}}\left|f^{\prime}(t)\right|^{q} d t\right)^{\frac{1}{q}}\right.$

$\left.+\sum_{k=0}^{\infty} \frac{\sigma(k)|w|^{k}}{\Gamma(\alpha+\rho k)}\left(\int_{\frac{a+b}{2}}^{b} \frac{(b-t)^{(\alpha+\rho k) p}}{(\alpha+\rho k)^{p}} d t\right)^{\frac{1}{p}}\left(\int_{\frac{a+b}{2}}^{b}\left|f^{\prime}(t)\right|^{q} d t\right)^{\frac{1}{q}}\right\}$

$=\|g\|_{[a, b], \infty}\left\{\sum_{k=0}^{\infty} \frac{\sigma(k)|w|^{k}}{\Gamma(\alpha+\rho k+1)}\left(\left.\frac{(t-a)^{\alpha p+\rho k p+1}}{\alpha p+\rho k p+1}\right|_{a} ^{\frac{a+b}{2}}\right)^{\frac{1}{p}}\left(\int_{a}^{\frac{a+b}{2}}\left|f^{\prime}(t)\right|^{q} d t\right)^{\frac{1}{q}}\right.$

$\left.+\sum_{k=0}^{\infty} \frac{\sigma(k)|w|^{k}}{\Gamma(\alpha+\rho k+1)}\left(-\left.\frac{(b-t)^{\alpha p+\rho k p+1}}{\alpha p+\rho k p+1}\right|_{\frac{a+b}{2}} ^{b}\right)^{\frac{1}{p}}\left(\int_{\frac{a+b}{2}}^{b}\left|f^{\prime}(t)\right|^{q} d t\right)^{\frac{1}{q}}\right\}$

$=\|g\|_{[a, b], \infty}\left\{\sum_{k=0}^{\infty} \frac{\sigma(k)|w|^{k}}{\Gamma(\alpha+\rho k+1)}\left(\frac{(b-a)^{\alpha p+\rho k p+1}}{2^{\alpha p+\rho k p+1}(\alpha p+\rho k p+1)}\right)^{\frac{1}{p}}\left(\int_{a}^{\frac{a+b}{2}}\left|f^{\prime}(t)\right|^{q} d t\right)^{\frac{1}{q}}\right.$

$\left.+\sum_{k=0}^{\infty} \frac{\sigma(k)|w|^{k}}{\Gamma(\alpha+\rho k+1)}\left(\frac{(b-a)^{\alpha p+\rho k p+1}}{2^{\alpha p+\rho k p+1}(\alpha p+\rho k p+1)}\right)^{\frac{1}{p}}\left(\int_{\frac{a+b}{2}}^{b}\left|f^{\prime}(t)\right|^{q} d t\right)^{\frac{1}{q}}\right\}$

$=\|g\|_{[a, b], \infty}\left\{\sum_{k=0}^{\infty} \frac{\sigma(k)|w|^{k}}{\Gamma(\alpha+\rho k+1)}\left(\frac{(b-a)^{\alpha+\rho k+\frac{1}{p}}}{2^{\alpha+\rho k+\frac{1}{p}}(\alpha p+\rho k p+1)^{\frac{1}{p}}}\right)\left(\int_{a}^{\frac{a+b}{2}}\left|f^{\prime}(t)\right|^{q} d t\right)^{\frac{1}{q}}\right.$

$\left.+\sum_{k=0}^{\infty} \frac{\sigma(k)|w|^{k}}{\Gamma(\alpha+\rho k+1)}\left(\frac{(b-a)^{\alpha+\rho k+\frac{1}{p}}}{2^{\alpha+\rho k+\frac{1}{p}}(\alpha p+\rho k p+1)^{\frac{1}{p}}}\right)\left(\int_{\frac{a+b}{2}}^{b}\left|f^{\prime}(t)\right|^{q} d t\right)^{\frac{1}{q}}\right\}$

$\leq\|g\|_{[a, b], \infty}(b-a)^{\alpha+1}{\underset{\mathcal{F}}{\rho, \alpha+1}}_{\rho, 1}^{\sigma_{1}}\left[|w|(b-a)^{\rho}\right]$

$\times\left\{\left(\frac{3\left|f^{\prime}(a)\right|^{q}+\left|f^{\prime}(b)\right|^{q}}{8}\right)^{\frac{1}{q}}+\left(\frac{\left|f^{\prime}(a)\right|^{q}+3\left|f^{\prime}(b)\right|^{q}}{8}\right)^{\frac{1}{q}}\right\}$.

Here we use

$$
\begin{aligned}
\int_{a}^{\frac{a+b}{2}}\left|\int_{a}^{t}(s-a)^{\alpha+\rho k-1} d s\right|^{p} d t & =\int_{\frac{a+b}{2}}^{b}\left|\int_{b}^{t}(b-s)^{\alpha+\rho k-1} d s\right|^{p} d t \\
& =\frac{(b-a)^{\alpha p+\rho k p+1}}{2^{\alpha p+\rho k p+1}(\alpha+\rho k)^{p}(\alpha p+\rho k p+1)}
\end{aligned}
$$




$$
\begin{aligned}
\int_{a}^{\frac{a+b}{2}}\left|f^{\prime}(t)\right|^{q} d t & \leq \frac{1}{b-a} \int_{a}^{\frac{a+b}{2}}\left[(b-t)\left|f^{\prime}(a)\right|^{q}+(t-a)\left|f^{\prime}(b)\right|^{q}\right] d t \\
& =(b-a) \frac{3\left|f^{\prime}(a)\right|^{q}+\left|f^{\prime}(b)\right|^{q}}{8}
\end{aligned}
$$

and

$$
\begin{aligned}
\int_{\frac{a+b}{2}}^{b}\left|f^{\prime}(t)\right|^{q} d t & \leq \frac{1}{b-a} \int_{\frac{a+b}{2}}^{b}\left[(b-t)\left|f^{\prime}(a)\right|^{q}+(t-a)\left|f^{\prime}(b)\right|^{q}\right] d t \\
& =(b-a) \frac{\left|f^{\prime}(a)\right|^{q}+3\left|f^{\prime}(b)\right|^{q}}{8} .
\end{aligned}
$$

Hence the inequality (2.7) is proved.

Remark 3. If we choose $\sigma(0)=1$ and $w=0$ in Theorem 6 , then the inequality (2.7) reduces to (1.12).

\section{ACKNOWLEDGEMENT}

This research is supported by Ordu University Scientific Research Projects Coordination Unit (BAP). Project Number: BY-1716

\section{REFERENCES}

[1] R. Agarwal, M.-J. Luo, and R. Raina, "On Ostrowski type inequalities," Fasciculi Mathematici, vol. 204, pp. 5-27, 2016, doi: 10.1515/fascmath-2016-0001.

[2] M. Awan, M. Noor, M. Mihai, and K. Noor, "Fractional Hermite-Hadamard inequalities for differentiable s-Godunova-Levin functions," Filomat, vol. 30, no. 12, pp. 3235-3241, 2016, doi: 10.2298/FIL1612235A.

[3] Z. Dahmani, "New inequalities in fractional integrals," Int. J. Nonlinear Sci., vol. 9, no. 4, pp. 493-497, 2010.

[4] Z. Dahmani, L. Tabharit, and S. Taf, "Some fractional integral inequalities," Nonlinear. Sci. Lett. A, vol. 1, no. 2, pp. 155-160, 2010.

[5] S. Dragomir and C. Pearce, Selected Topics on Hermite-Hadamard Inequalities and Applications. RGMIA Monographs, Victoria University, 2000.

[6] L. Fejér, "Uber die fourierreihen, ii," Math. Naturwiss Anz. Ungar. Akad. Wiss, Hungarian, vol. 24, pp. 369-390, 1906.

[7] I. Işcan, "Hermite-Hadamard-Fejer type inequalities for convex functions via fractional integrals," Stud. Univ. Babes-Bolyai Math., vol. 60, no. 3, pp. 355-366, 2015.

[8] M. Noor, K. Noor, and M. Awan, "Fractional Hermite-Hadamard inequalities for two kinds of s-preinvex functions," Nonlinear Sci. Lett. A, vol. 8, no. 1, pp. 11-24, 2017.

[9] R. Raina, "On generalized Wright's hypergeometric functions and fractional calculus operators," East Asian Math. J., vol. 21, no. 2, pp. 191-203, 2005.

[10] M. Sarıkaya, E. Set, H. Yaldız, and N. Başak, "Hermite-Hadamard's inequalities for fractional integrals and related fractional inequalities," Math. Comput. Model., vol. 57, no. 9, pp. 24032407, 2013, doi: 10.1016/j.mcm.2011.12.048.

[11] E. Set, I. İşcan, M. Sarıkaya, and M. Özdemir, "On new inequalities of Hermite-Hadamard-Fejér type for convex functions via fractional integrals," Appl. Math. Comput., vol. 259, pp. 875-881, 2015, doi: 10.1016/j.amc.2015.03.030. 
[12] E. Set, M. Özdemir, and M. Sarkkaya, "Inequalities of Hermite-Hadamard type for functions whose derivatives absolute values are m-convex," AIP Conference Proceedings, vol. 1309, no. 861, 2010, doi: 10.1063/1.3525219.

[13] E. Set, M. Sarıkaya, M. Özdemir, and H. Yıldırım, "The Hermite-Hadamard's inequality for some convex functions via fractional integrals and related results," J. Appl. Math. Statis. Inform., vol. 10, no. 2, pp. 69-83, 2014, doi: 10.2478/jamsi-2014-0014.

Authors' addresses

\section{Erhan Set}

Ordu University, Department of Mathematics, Faculty of Science and Arts, Ordu, Turkey

E-mail address: erhanset@yahoo.com

\section{Barış Çelik}

Ordu University, Department of Mathematics, Faculty of Science and Arts, Ordu, Turkey

E-mail address: bariscelik15@hotmail.com 